OPEN

SUBJECT AREAS:

NANOSCIENCE AND

TECHNOLOGY

OPTICAL PROPERTIES AND DEVICES

Received

28 August 2013

Accepted

8 November 2013

Published

25 November 2013

Correspondence and requests for materials should be addressed to V.A.K. (victi81@mail.

\section{Carbon nanowalls: the next step for physical manifestation of the black body coating}

V. A. Krivchenko', S. A. Evlashin' ', K. V. Mironovich', N. I. Verbitskiy'2,3, A. Nefedov', C. Wöll', A. Ya. Kozmenkova' ${ }^{2}$, N. V. Suetin ${ }^{1,8}$, S. E. Svyakhovskiy' , D. V. Vyalikh' ${ }^{6}$, A. T. Rakhimov' , A. V. Egorov ${ }^{7}$ \& L. V. Yashina ${ }^{7}$

'D.V. Skobeltsyn Institute of Nuclear Physics, M.V. Lomonosov Moscow State University, Leninskie Gory, 1/2, 119991 Moscow, Russia, ${ }^{2}$ Department of Materials Science, M.V. Lomonosov Moscow State University, Leninskie Gory, 1/3, 119991 Moscow, Russia, ${ }^{3}$ Faculty of Physics, University of Vienna, Strudlhofgasse 4, A-1090 Wien, Austria, ${ }^{4}$ Institut für Funktionelle Grenzflächen (IFG), Karlsruher Institut für Technologie (KIT), Hermann-von-Helmholtz-Platz 1, D-76344 Eggenstein-Leopoldshafen, Germany, ${ }^{5}$ Department of Physics, M.V. Lomonosov Moscow State University, Leninskie Gory, 1/2, 119991 Moscow, Russia, Institut für Festkörperphysik, TU Dresden, Mommsenstrasse 13, D-01069 Dresden, Germany, ${ }^{7}$ Department of Chemistry, M.V. Lomonosov Moscow State University, Leninskie Gory, 1/3, 119991 Moscow, Russia, ${ }^{8}$ Skolkovo Institute of Science and Technology, Skolkovo 143025 Skolkovo, Russian Federation.

The optical properties of carbon nanowall (CNW) films in the visible range have been studied and reported for the first time. Depending on the film structure, ultra-low total reflectance up to $0.13 \%$ can be reached, which makes the CNW films a promising candidate for the black body-like coating, and thus for a wide range of applications as a light absorber. We have estimated important trends in the optical property variation from sample to sample, and identified the presence of edge states and domain boundaries in carbon nanowalls as well as the film mass density variation as the key factors. Also we demonstrated that at much lower film thickness and density than for a carbon nanotube forest the CNWs yield one order higher specific light absorption.

P he black body, which is a simple physical model well known from the school optics course, remains highly demanded for a wide range of implementations and solutions for many technological issues. Apparently, practical preparation of the black body-like materials will bring benefits to many fields such as sensing, radiometric and energy harvesting. Nowadays, among all materials a forest of vertically aligned single-walled carbon nanotubes behaves in a manner most similar to that of the black body ${ }^{1-3}$. This material can absorb light almost perfectly in a very wide spectral range $(0.2-200 \mu \mathrm{m})$. The corresponding total reflectance is found to be in the range of $0.045 \%-0.8 \%$ for the visible light ${ }^{2,3}$.

Here, we report a new candidate for the black body-like coating, carbon nanowall (CNW) films. The CNW film is a material composed of a dense array of micron-size flakes with layered graphite-like structure and dominating vertical orientation and chaotic lateral displacement with hundreds of nm mean spacing. Thickness of each flake may vary from several graphene layers to tens of nanometers which is the reason for term nanowall. The most widely used method for the CNW film fabrication is plasma enhanced chemical vapour deposition with different ways of plasma activation ${ }^{4,5}$. This material has already found a variety of applications such as an electron field emitters $^{6}$, a catalyst support ${ }^{7}$, and a template for a different nanostructured materials ${ }^{8,9}$. In present work we report for the first time that the $\mathrm{CNW}$ films demonstrate optical properties in the visible range that are close to those of a perfect light absorber. Compared to a nanotube forest with a thickness of hundreds of microns, the CNW films are much thinner (only several microns) and hence specific light absorption characteristics (normalized by the film mass per substrate area) are essentially higher. One of the advantages is that carbon nanowalls can be grown without any catalysts on a variety of substrates including silicon, different metals and other carbon materials ${ }^{4,5,7,10}$. The absence of catalyst (usually $3 d$-metals) makes this material suitable for high-temperature applications. Due to H-termination the CNW film surface is hydrophobic and very stable against structural degradation, especially in a humid environment. These advantages may result in broader applications of carbon nanowalls as extremely dark thin coating, for instance, for bolometers. 
The present paper includes the discussion of the CNW film structure effect on the optical properties. We have found that optical absorption is inversely proportional to mean carbon nanowall linear size and proportional to the film density, i.e. to amount of carbon nanowalls per unit of substrate area. Based on obtained results we assume by analogy with graphene nanoribbons ${ }^{11}$ that presence of edge states in carbon nanowalls is one of the key factors determining high light absorption in the visible range.

\section{Results}

For our studies we have chosen CNW films (CF1-CF5) obtained at different growth conditions providing strongly different film morphology, i.e. various combinations of structural parameters. The structure of tested CNW films is illustrated in Fig. 1. We quantify the morphology with the following structural parameters: average carbon nanowall linear dimension (based on the top view SEM images), film density (the number of carbon nanowalls per unit of substrate area) and the overall film thickness as shown in Table 1. It should be noted that CNW films of different morphology are reproducibly obtained by adjusting the corresponding growth conditions ${ }^{4,12}$. This issue, however, is beyond of our consideration.

As it is clearly seen from Fig. $1 \mathrm{a}-\mathrm{b}$ the structure of samples CF1 and CF2 contains smaller nanowalls besides the major carbon nanowall network. These smaller carbon nanowalls emerge due to secondary nucleation and we refer to such structures as the secondary carbon nanowalls, or SCNWs. This phenomenon is not considered further and will be described in details in our next article.

Samples CF1 and CF2 have approximately same thickness of about $3 \mu \mathrm{m}$. However, film CF1 may be characterized by higher SCNW coverage than those of film CF2. This leads to higher carbon nanowall surface density $\left(23 \mu \mathrm{m}^{-2} / 13 \mu \mathrm{m}^{-2}\right.$ for CF1/CF2) and to decrease in calculated mean carbon nanowall linear size $(310 \mathrm{~nm} /$ $370 \mathrm{~nm}$ for CF1/CF2).

Fig. 2a shows a low-resolution cross-sectional TEM view of an individual carbon nanowall with SCNWs on its surface. Fig. $2 b$ demonstrates high resolution TEM image of an individual SCNW, where its multilayered structure is clearly visible. Both major carbon nanowall and the SCNW have higher thickness at their origin than at the end.

Films CF3, CF4 and CF5 are composed of carbon nanowalls with different height (film thickness) and mean linear size. No SCNWs were observed for these samples (Fig. 1c-e). Thus film CF3 has both the maximal thickness and the maximal mean carbon nanowall linear dimension among the samples, $5 \mu \mathrm{m}$ and $900 \mathrm{~nm}$, respectively and a relatively low carbon nanowall surface density of $3 \mu \mathrm{m}^{-2}$. In turn CF4 and CF5 have thickness of about $2-2.5 \mu \mathrm{m}$ and some intermediate carbon nanowall surface density of $7 \mu \mathrm{m}^{-2}$.

General structural characterisation of the obtained CNW films were performed using Raman, near edge X-ray absorption fine structure (NEXAFS) and high-resolution X-ray photoelectron spectroscopy (XPS).

A Raman spectrum for a typical CNW film is presented in Fig. 3a. The corresponding spectral parameters for the films grown at different discharge conditions are shown in Table 1. The Raman spectrum includes a $G$ mode usually observed for the graphite-based materials, distinguished $D$ and $D^{\prime}$ modes that could be associated with the lattice defects of different nature, and $2 D$ mode that corresponds to the second order of the $D$ mode. The $I(D) / I(G)$ ratio, which is related to the crystal imperfection of a graphene sheet, varies from 0.2 to 1.5. It is known that plasma grown carbon nanowalls contain a lot of structural defects and conditionally may be described as an agglomeration of nanometer-size graphene-like domains ${ }^{13}$. Thus appearance of $\mathrm{D}$ mode may be associated with presence of both carbon nanowall and domain edges. In our case domain structure of studied carbon nanowalls may be seen in Fig. 2c. Taking into account $I(D) / I(G)$ ratios shown in Table 1 estimated domain linear size varies from $3 \mathrm{~nm}$ to $22 \mathrm{~nm}^{14}$. Moreover it was shown that in case of graphene $I(D) / I\left(D^{\prime}\right)$ ratio does not depend on the defects concentration but is sensitive to the type of defects ${ }^{15}$. For our CNW films, it varies between 1.8 and 3.3 which also may indicate presence of boundary-like defects in a carbon nanowall structure.

We found an inverse correlation between the mean linear size of the carbon nanowalls and the $I(D) / I(G)$ ratio (see Table 1 ). Thus, the highest D mode peak is observed for the sample CF1 while sample CF3 has the minimal boundary-like defect density. The CF4 and CF3 films differ by their $I(D) / I(G)$ value, which may be attributed to different density of the domains within an individual carbon nanowall.

Appearance of $2 D$ mode does not depend on presence of the defects, however its position, FWHM and shape are sensitive to the layer stacking character, namely, turbostratic stacking or ABAB (like in graphite $)^{16,17}$. For carbon nanowalls the $2 D$ peak is symmetrical

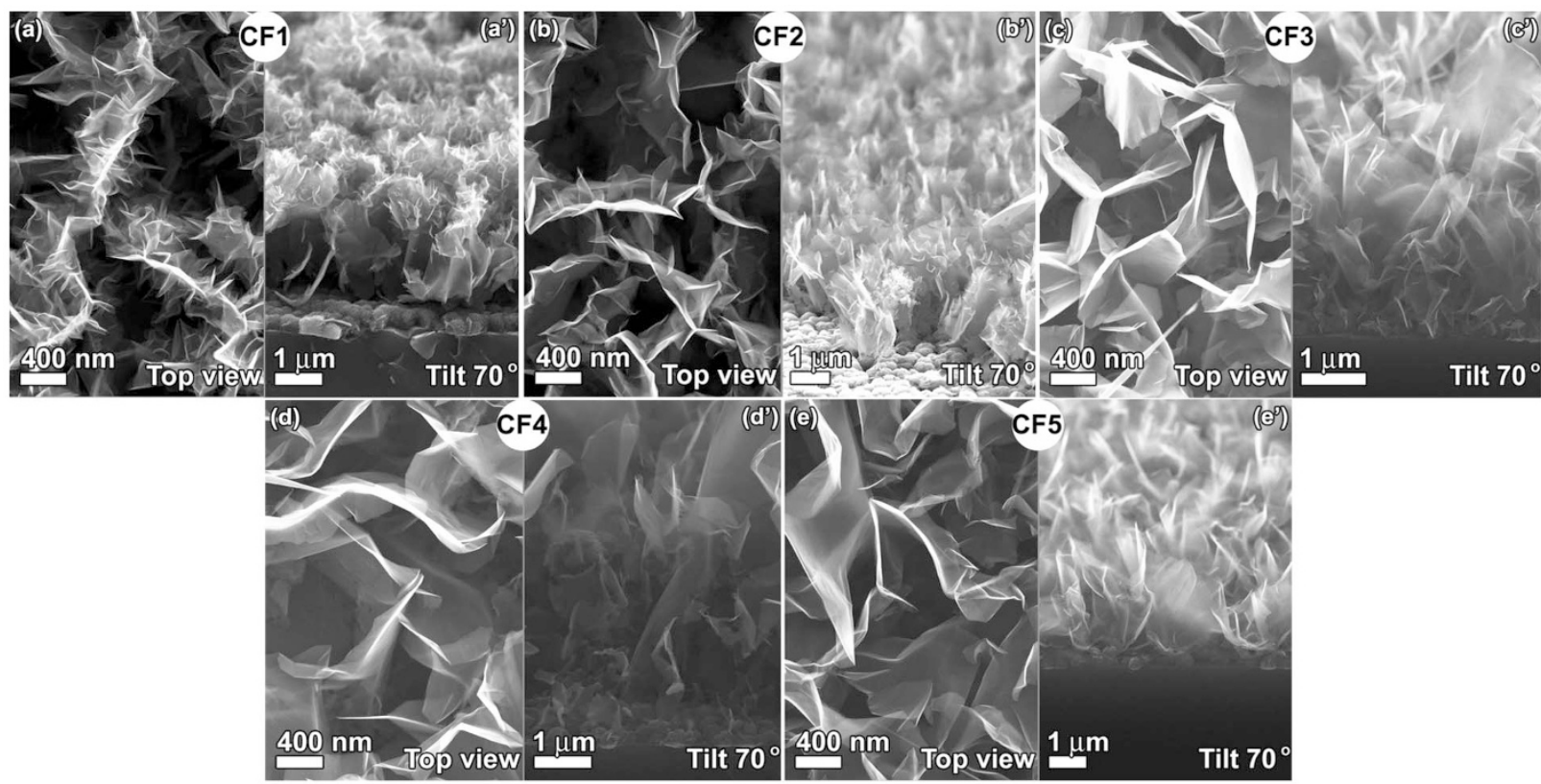

Figure 1 SEM images of top view (a-e) and side view $\left(\mathrm{a}^{\prime}-\mathrm{e}^{\prime}\right)$ of the CNW films on a Si (100) substrate. 
Table 1 | Summary of the structural and Raman spectral parameters of the CNW films

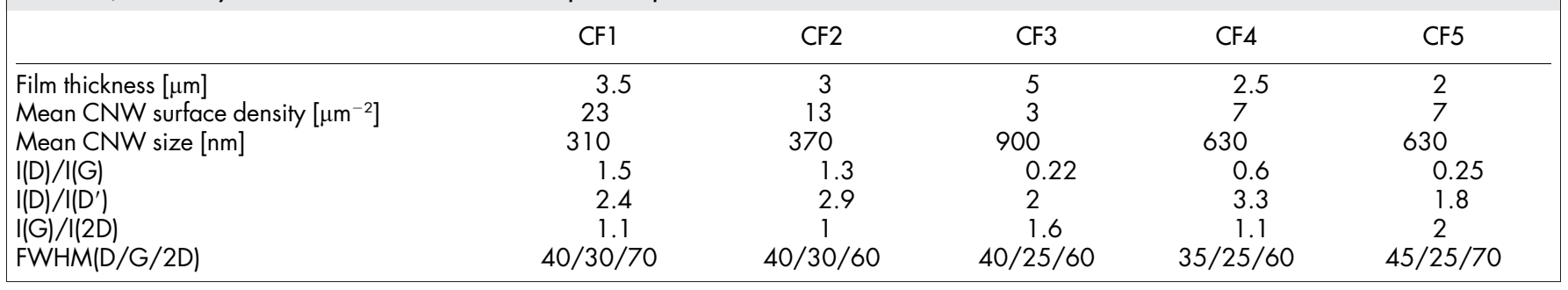

and relatively broad, thus indicating the presumably turbostratic structure.

It should be noted that FWHMs of the D, G, and 2D modes are almost similar for all samples. The line broadening is sensitive to crystal imperfections and the amorphization degree ${ }^{14}$. This means that carbon nanowall (domain) imperfection with both point defects and other types of imperfections is approximately the same in all samples under discussion.

A typical XPS C1s spectrum for a CNW sample (Fig. 3b) includes a dominating peak of the $s p^{2}$-hybridized carbon, which is slightly broader than that for graphite and essentially broader than dominating peak for epitaxially grown graphene monolayer ${ }^{18}$. Additional high-energy peak is related to the $s p^{3}$-hybridized carbon atoms located near defect sites or edges.

The C $K$-edge NEXAFS spectra for a typical sample without SCNWs acquired at different X-ray incidence angles show high intensive $\pi^{*}$ - and $\sigma^{*}$-resonances and broad peaks in the range of 292-320 eV, which correspond to transitions from the $\mathrm{C} 1 \mathrm{~s}$ level to unoccupied $\sigma$ states. The NEXAFS spectra show slight angular dependence corresponding to the mean deflection of the carbon nanowalls equal to $30^{\circ}$ from normal to the substrate surface.

Optical properties such as reflectance in the visible range of 400$1000 \mathrm{~nm}$ and its angular dependence were studied for a number of the CNW films described in Table 1. Fig. 4a shows the optical reflectance of the CNW films at normal incidence and a detection angle of $45^{\circ}$. It is remarkable that for sample CF1 the reflectance reaches up to $0.003 \%$, while reflectance for other samples is $2-3$ times higher. The angular dependence of the specular reflectance obtained at $500 \mathrm{~nm}$ is shown in Fig. 4b. Sample CF1, which has the minimal reflectance, shows no noticeable angular dispersion of the specular reflectance typical for the nanotube forest ${ }^{1}$. The hemispherical (total) reflectance for the CNW films is illustrated in Fig. 4c. The minimal value of the total reflectance reaches the value of $0.13 \%$ for the sample CF1.

Our study of a CNW film transparency without silicon (or any other) substrate revealed no detectable signal penetrated through the film (experimental details are described in Supplementary Information file). This result indicates that light absorption may be estimated as $100 \%$ minus the light reflectance and amounts to $99.997 \%$ at normal incidence and detection angle of $45^{\circ}$ and $99.87 \%$ in case of the total reflectance measurements. It should be noted that films that are less than $1 \mu \mathrm{m}$ thick are significantly transparent, and this may be considered as a limit for CNW films acting as an effective light absorber.

Summing up, the optical properties of the carbon nanowalls in the visible range make this material attractive for many applications as an extremely dark thin coating.

\section{Discussion}

Now let us address the main reasons providing high optical absorbance (low reflection) of a relatively thin carbon nanowall films. The light absorbance by a coating is determined by two main factors: efficiency of reflectance at a film-media interface and efficiency of light absorption inside a film. Evidently, the interface morphology plays crucial role in the light propagation. For example, antireflection coatings composed of submicron-size Si structures ${ }^{19-21}$ show much lower optical reflectance when composed of Si cones as compared to those made of $\mathrm{Si}$ whiskers ${ }^{21}$. The authors used the gradient refractive index model to describe the optical properties ${ }^{19,20}$. For cone structures, there is non-uniform filling factor (relative volume of active material in a total volume of a coating) that varies with the film thickness, and the effective refractive index increases from the coating surface to the substrate. In that context, light can penetrate through a film without any significant phase retardation. We see here an analogy with our material. According to our TEM and SEM data, the CNW films also have non-uniform in-depth density. We assume that the films may be characterised by a variable refractive index (variable filling factor) that leads to the lower light reflection.

However, not only light reflection but also its transmission should be avoided since light may reflect from a film-substrate interface and penetrate through a material back to the ambient.
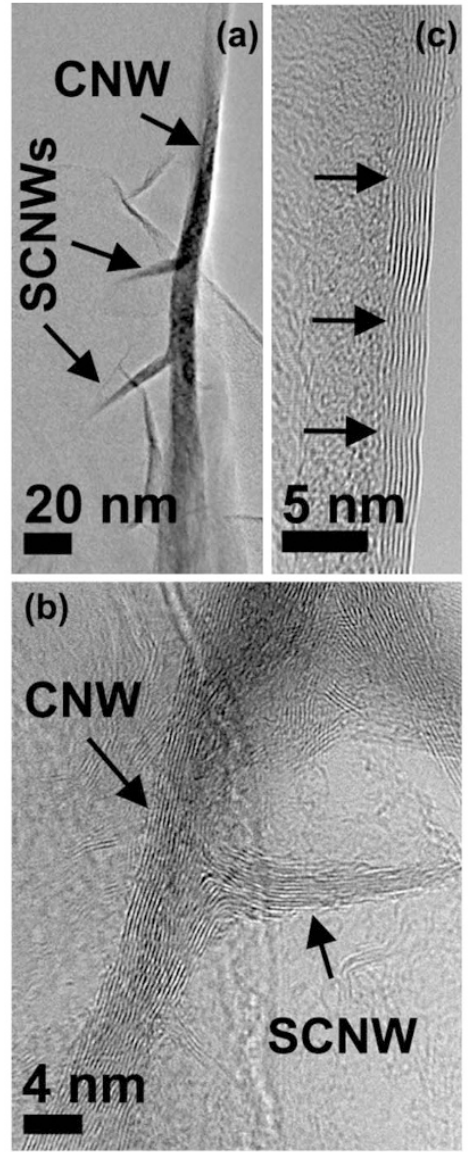

Figure $2 \mid$ Low-resolution TEM image of an individual carbon nanowall with SCNWs (a), high-resolution TEM image of SCNW (b) and highresolution TEM image of a carbon nanowall fragment (c): domain boundaries are marked by arrows. 

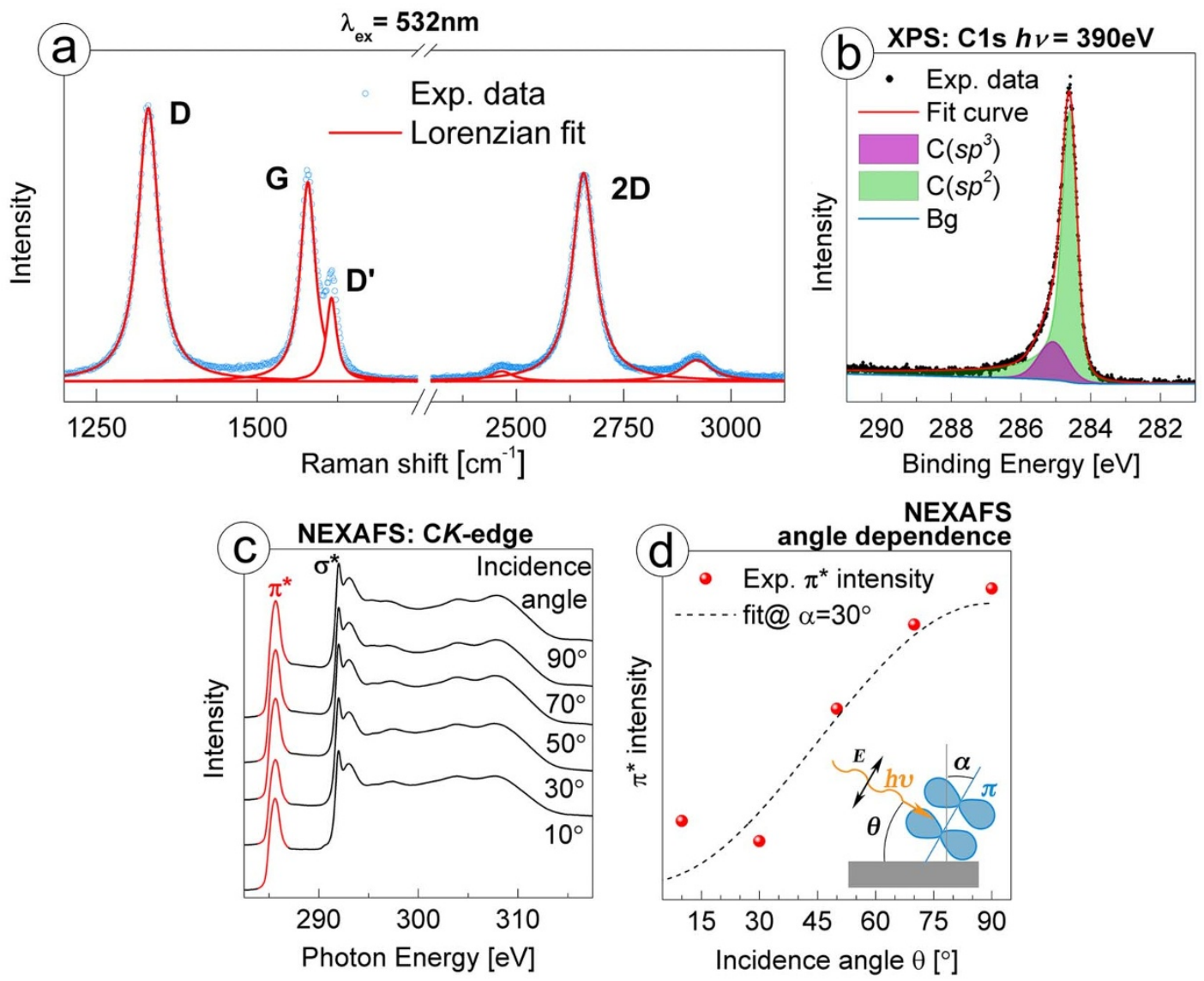

Figure 3 Typical Raman spectrum of a CNW film obtained at a laser wavelength of $532 \mathrm{~nm}$ and a radiation power of $30 \mathrm{~mW}$ (a); spectrum of film CF2 is demonstrated as an example, C1s photoemission (b) and NEXAFS spectra (c) for a CNW film obtained at different angles, angular dependence of the $\pi^{*}$-resonance intensity from the NEXAFS spectra (d): the insert shows the geometry of the experiment and the $\pi$-system displacement relative to the surface normal, $\alpha=30^{\circ}$ corresponds to the mean deviation of the carbon nanowall from the surface normal.

Optical absorption of graphene-type materials is determined by direct interband transition of $\pi$-electrons. In case of large-scale graphene the absorption coefficient is proportional to $\pi \alpha^{22}$. For fewlayer graphene it is proportional to the number of layers $(N \pi \alpha)$. The reflection coefficient of a graphene single layer is very low and increases with the number of layers ${ }^{23}$.

The decrease of the lateral dimensions of graphene flake gives rise to essential contribution of edges into the electronic structure. Thus, theoretical analysis of carbon nanoribbons demonstrates appearance of new states localized near the Fermi level ${ }^{11}$ due to edge states. The density of such electronic states decays exponentially from the nanoribbon edge to its center. The appearance of such intraband electronic states is not associated with the structure of bulk graphite or the dangling bonds at the crystal edge and may be explained by certain features of the $\pi$ electron system of a nanosized structure. It was shown that increase in the crystal linear size leads to a decrease in the density of the electronic states induced by the edges ${ }^{11}$. Study of graphene nanoribbon optical properties revealed the edge states are essential since the intraband electronic states are involved in the light absorption process ${ }^{24-26}$.

In this respect the carbon nanowalls with domain structure may be considered as an intermediate case between nanoribbons and large scale few-layered graphene. We suppose that similar to nanoribbons the large quantity of edges in carbon nanowalls influence their electronic structure. By varying the liner dimensions of the carbon nanowalls (and the domains) it is possible to modify the electronic properties from those typical for nanoribbons to those typical for large scale graphene.
This concept may explain the experimental optical reflection (absorption) data obtained for different CNW samples. The best properties were observed for the film CF1, which is the densest film and is composed of carbon nanowalls with the minimal size. In its turn, an increase in the carbon nanowall linear size or decrease in their surface density (sample CF3) leads to decrease in the total density of the edge states. As a consequence, a decrease in the light absorption (increase in reflectance) is observed. Moreover variation in domain liner size at constant nanowall surface density and nanowall size leads to variation in optical properties as well. It is clearly seen from comparing structural and optical features of samples CF4 and CF5.

It should be mentioned that nothing is known about the possible effect of the atomic structure (layer stacking) and the surface termination (like hydrogen termination typical for carbon nanowalls obtained under the glow discharge) on the optical properties. As these factors influence the electronic properties of carbon materials, their role in the optical behaviour may be essential.

Behaviour of reflectance of the s-and p-polarised light is shown in Fig. 4 d. It is clearly seen that optical properties of the CNW film do not depend on the incident light polarisation. This is in line with generally chaotic displacement of the carbon nanowalls.

Based on the presented results, we performed estimation of the CNW film specific absorption (absorption per unit of areal mass density) and compared it with the data for the CNT forest ${ }^{2}$. Specific absorption of the CNW film achieves up to $6600 \mathrm{~g}^{-1} \mathrm{~cm}^{2}$ in the visible range, which is almost one order higher than the corresponding values for the CNT forest (more details are provided in 

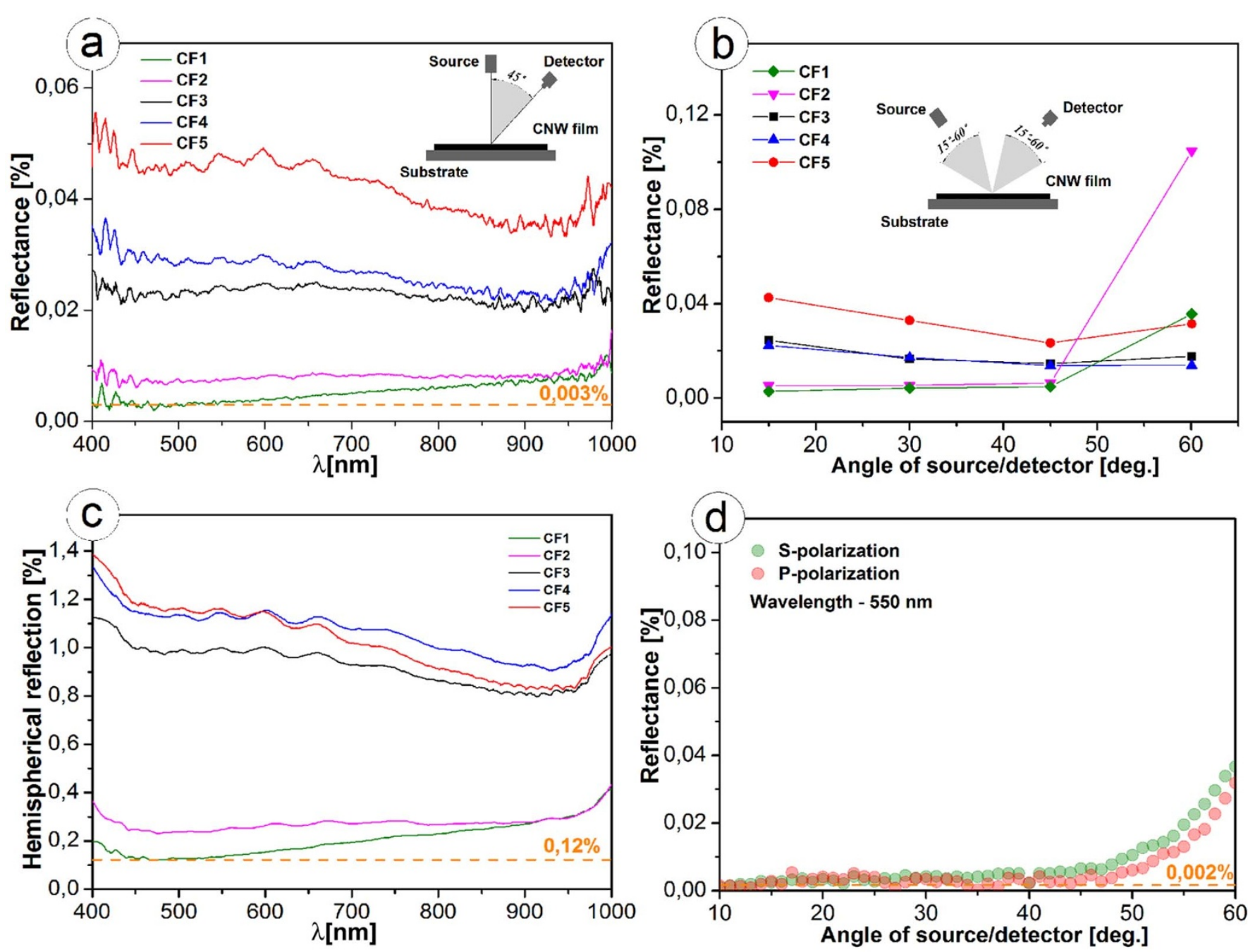

Figure $4 \mid$ Reflectance at normal incidence (a), angle dependence of the specular reflectance (b), and hemispherical (total) reflectance of the CNW films at normal light incidence (c), specular reflectance of s- and p-polarized light for film CF1 (d): angles of both detector and light source were varied in the range of $10-60^{\circ}$.

the Supplementary Information, Table S2). The underlying reason is in different ways of light absorption in these two cases. For the nanotubes high absorption is provided with long optical path and multiple reflections/absorption, e.g. in cavities between relatively long oriented nanotubes. Therefore, the optical properties of the nanotube forest depend on their length and surface density and display polarisation anisotropy ${ }^{27}$. In the case of carbon nanowalls the key factor is the edge state density which is higher for lower nanowall dimensions.

A carbon nanowall-based dark coating can find a wide array of applications. One of them is a bolometer absorber. Here we can utilize much lower film thickness than for the carbon nanotube forest. In addition, the CNW films are very stable against structural degradation in humid media like ambient atmosphere due to the hydrophobic nature of an as-grown carbon nanowall surface as a result of $\mathrm{H}$-termination ${ }^{4}$. This stability is of great importance for high temperature applications.

In summary, we report first observations of the optical properties of carbon nanowall films in the visible range. It was shown that the CNW films possess outstanding optical properties, such as extremely low reflectance and high specific absorption. The CNW films demonstrate optical properties comparable to those of the carbon nanotube forest, which has so far been considered as the best approximation of the black body. The specific absorption of the CNW films actually exceeds that of the CNT forest. It is remarkable that the total reflectance of the CNW film can reach up to $0.13 \%$. In addition, we groped certain important trends in the structure/optical property dependence for the carbon nanowalls. The major factors are found to be structural imperfection, presence of edge states and surface density of the carbon nanowalls over the substrate.

\section{Methods}

The films were grown on polished $p$-doped $460 \mu \mathrm{m}$ thick Si (100) wafers with an area of $1 \mathrm{~cm}^{2}$. All substrates were preliminary ultrasonically treated in a diamond powder suspension for $5 \mathrm{~min}$ to assist the carbon film nucleation site formation. Further on, the substrates were washed in distilled water and dried. The CNW films were grown in the plasma of dc glow discharge in a mixture of hydrogen and methane. Substrates were placed on molybdenum anode. The working mixture pressure was 50 Torr at the moment of discharge ignition and was gradually increased up to 150 Torr. Further growth conditions are listed in Table S1 in the Supporting Information file. The samples were studied by means of SEM (Carl Zeiss Supra 40 system), TEM (model JEM 2100F (UHR/Cs) with acceleration voltage - $200 \mathrm{kV}$ ), and Raman spectroscopy (Renishaw InVia Reflex spectrometer using a $514 \mathrm{~nm}$ wavelength laser with a power of $30 \mathrm{~mW}$ ). XPS and NEXAFS measurements were performed at RGBL and HESGM beamlines at BESSY II synchrotron facility (Berlin, Germany).The samples were preliminary annealed at $400^{\circ} \mathrm{C}$ for $30 \mathrm{~min}$ to remove the adventitious pollutions. Optical measurements in a range of $400-1000 \mathrm{~nm}$ were performed with use of Avesta 100MF spectrometer with rotating polarizer and fiber input. Tungsten halogen lamp was used as a light source. When electric filed vector of incident light is perpendicular (parallel) to the plane of incidence it corresponds to s-polarization (ppolarization). The CNW film mass was estimated based on the results of gravimetric measurements of the substrates before and after the film deposition by means of digital analytical micro balance AND HM-202 (precision $0.01 \mathrm{mg}$ ). 
1. Mizuno, K. et al. A black body absorber from vertically aligned single-walled carbon nanotubes. Proc. Natl. Acad. Sci. 106, 6044-6047 (2009).

2. Yang, Z.-P., Ci, L., Bur, J. A., Lin, S.-Y. \& Ajayan, P. M. Experimental observation of an extremely dark material made by a low-density nanotube array. Nano Lett. 8(2), 446-451 (2008).

3. Kaul, A. B., Coles, J. B., Eastwood, M., Green, R. O. \& Bandaru, P. R. Ultra-high optical absorption efficiency from the ultraviolet to the infrared using multiwalled carbon nanotube ensembles. Small 9, 1058-1065 (2013).

4. Krivchenko, V. A. et al. Evolution of carbon film structure during its catalyst-free growth in the plasma of direct current glow discharge. Carbon 50, 1477-1487 (2012).

5. Kondo, S. et al. Initial growth process of carbon nanowalls synthesized by radical injection plasma-enhanced chemical vapor deposition. J. Appl. Phys. 106, 094302 (2009).

6. Malesevic, A. et al. Field emission from vertically aligned few-layer graphene. J. Appl. Phys. 104, 084301 (2008).

7. Podlovchenko, B. I. et al. Specific features of the formation of $\mathrm{Pt}(\mathrm{Cu})$ catalysts by galvanic displacement with carbon nanowalls used as support. Electrochimica Acta 76, 137-144 (2012).

8. Krivchenko, V. A. et al. Carbon nanowalls decorated with silicon for lithium-ion batteries. Carbon 50, 1438-1442 (2012).

9. Wang, H., Su, Y., Chen, S. \& Quan, X. Growth of tungsten oxide on carbon nanowalls templates. Materials Research Bulletin 48(13), 1304-1307 (2013).

10. Wang, H.-X., Jiang, N., Zhang, H. \& Hiraki, A. Growth of a three-dimensional complex carbon nanoneedle electron emitter for fabrication of field emission device. Carbon 48, 4483-4488 (2010).

11. Nakada, K., Fujita, M., Dresselhaus, G. \& Dresselhaus, M. S. Edge state in graphene ribbons: Nanometer size effect and edge shape dependence. Phys. Rev. B. 54(24), 17954-17961 (1996)

12. Krivchenko, V. et al. Influence of the growth temperature on structural and electron field emission properties of carbon nanowall/nanotube films synthesized by catalyst-free PECVD. J. Mater. Chem. 22, 16458-16464 (2012).

13. Kobayashi, K. et al. Nanographite domains in carbon nanowalls. J. Appl. Phys. 101, 094306 (2007).

14. Ferrari, A. C. \& Robertson, J. Interpretation of Raman spectra of disordered and amorphous carbon. Phys. Rev. B. 61(20), 14095-14107 (2000)

15. A. Eckmann, A. et al. Probing the nature of defects in graphene by raman spectroscopy. Nano Lett. 12, 3925-3930 (2012).

16. Lenski, D. R. \& Fuhrer, M. S. Raman and optical characterization of multilayer turbostratic graphene grown via chemical vapor deposition. J. Appl. Phys. 110, 013720 (2011).

17. Faugeras, C. et al. Few-layer graphene on $\mathrm{SiC}$, pyrolitic graphite, and graphene: A Raman scattering study. Appl. Phys. Lett. 92, 011914 (2008).

18. Grüneis, A., Kummer, K. \& Vyalikh, D. V. Dynamics of graphene growth on a metal surface: a time-dependent photoemission study. New J. Phys. 11, 073050 (2009).

19. Ravipati, S., Shieh, J., Ko, F.-H., Yu, C.-C. \& Chen, H.-L. Ultralow reflection from -Si nanograss/Si nanofrustum double layers. Adv. Mater. 25(12), 1724-8 (2013).
20. Huang, Y. F. et al. Improved broadband and quasi-omnidirectional anti-reflection properties with biomimetic silicon nanostructures. Nat. Nanotechnol. 2(12), 770-4 (2007).

21. Zhu, J. et al. Optical absorption enhancement in amorphous silicon nanowire and nanocone arrays. Nanoletters 9(1), 279-282 (2009).

22. Nair, R. R. et al. Fine structure constant defines visual transparency of graphene. Science 320, 1308 (2008).

23. Casiraghi, C. et al. Rayleigh imaging of graphene and graphene layers. Nanoletters 7(9), 2711-2717 (2007).

24. Hsu, H. \& Reichl, L. E. Selection rule for the optical absorption of graphene nanoribbons. Phys. Rev. B. 76, 045418 (2007).

25. Gundra, K. \& Shukla, A. Theory of the electro-optical properties of graphene nanoribbons. Phys. Rev. B. 83, 075413 (2011).

26. Sasaki, K., Kato, K., Tokura, Y., Oguri, K. \& Sogawa, T. Theory of optical transitions in graphene nanoribbons. Phys. Rev. B. 84, 085458 (2011).

27. Murakami, Y., Einarsson, E., Edamura, T. \& Maruyama, S. Polarization dependence of the optical absorption of single-walled carbon nanotubes. Phys. Rev. Lett. 94, 087402 (2005).

\section{Acknowledgments}

This work was partially supported by the Russian-German Laboratory at BESSY and by «Nanomicro Program» (KIT), grant MK-4994.2012.2 and GC No 14.516.11.0099. We thank Anton Stepanov (MSU) for support in SEM measurements.

\section{Author contributions}

V.A.K., S.A.E., L.V.Y., N.V.S. and R.A.T. designed this study and wrote the manuscript, K.V.M. synthesized the CNW films, S.E.S. performed optical measurements, A.Ya.K. performed Raman measurements, A.V.E. performed TEM study of the samples, N.I.V. A.N., C.W. and D.V.V. were responsible for XPS and NEXAFS measurements and analysis of obtained data.

\section{Additional information}

Supplementary information accompanies this paper at http://www.nature.com/ scientificreports

Competing financial interests: The authors declare no competing financial interests.

How to cite this article: Krivchenko, V.A. et al. Carbon nanowalls: the next step for physical manifestation of the black body coating. Sci. Rep. 3, 3328; DOI:10.1038/srep03328 (2013)

This work is licensed under a Creative Commons AttributionNonCommercial-ShareAlike 3.0 Unported license. To view a copy of this license, visit http://creativecommons.org/licenses/by-nc-sa/3.0 